\title{
La salud ergonómica del docente de una escuela militar en la región Amazónica
}

The ergonomic health of the teacher of a military school in the Amazon region

A saúde ergonômica do professor de uma escola militar na região Amazônica

Wesley Gomes Feitosa ${ }^{1}$, Elder Oliveira da Silva ${ }^{2 *}$, Suelen dos Santos Ferreira ${ }^{3}$, Pasionaria Rosa Ramos Ruiz Diaz ${ }^{4}$, Eliezer de Araújo ${ }^{5}$, José Maurício Diascânio .

\section{RESUMEN}

Objetivos: Describir la salud ergonómica de los docentes de una escuela militar en la Amazonia, ya que el trabajo desempeñado por este ocasiona interferencias en la integridad psicofisiológica del trabajador. Método: Se aplicó el instrumento de evaluación de fatiga utilizado por la Ergo Asesoría y Consultoría en Salud del Trabajador, validada por Couto, utilizado en las más diversas prácticas ocupacionales. Este instrumento mide el nivel de desconformidad del profesor al principio y al final del horario de trabajo Fue aplicado también el instrumento RULA. Resultados: Las mayores molestias presentadas fueron estrés 37\% (23), dolor muscular 32\% (20) y fatiga $31 \%$ (19). Al inicio de su jornada laboral, las mayores molestias relatadas fueron clasificadas como factores de intensidad uno (1); caracterizadas por la ausencia de dolores en los brazos o puños de las manos, ausencia de dolor en las costillas y productividad normal. Al finalizar la jornada, las mayores molestias referidas fueron factores con intensidad tres (3) a cuatro (4), tales como dolores en las costillas, dolores en las manos y puños del brazo dominante y el factor nervioso. Conclusión: El proceso de trabajo docente presenta un riesgo ergonómico moderado, siendo trabajo estresante, por lo que se requiere de adecuaciones para mejorar la calidad de vida y de trabajo del docente en la escuela.

Palabras clave: Ergonomía, Enseñanza, Análisis ergonómico.

\section{ABSTRACT}

Objectives: To describe the ergonomic health of teachers of a military school in the Amazon region, since the work done by this cause interferes in the psychophysiological integrity of the worker. Method: The instrument used to evaluate fatigue used by Ergo Assessoria e Consultoria em Saúde do Trabalhador, validated by Couto, was used in the most diverse occupational practices. This instrument measures the teacher's level of discomfort at the beginning and end of working hours. Results: The largest reported nonconformities were $37 \%$ stress (23), muscle pain $32 \%$ (20) and fatigue 31\% (19). At the beginning of the workday, the highest reported nonconformities were classified as intensity factors one (1), characterized by the absence of pain in the arms or wrists of the hands, absence of pain in the ribs and normal productivity. At the end of the day, the major reported nonconformities were intensity factors of three (3) to four (4), such as pain in the ribs, pain in the hands and wrists of the dominant arm and the nervous factor. Conclusion: The work process presents a moderate ergonomic risk, the work is stressful and requires adjustments to improve the quality of life and the teaching work of the school.

Keywords: Ergonomics, Teaching, Ergonomic analysis.

1,3,5 Universidad Columbia del Paraguay. Asunción - Paraguay.

2 Facultad de Ciencias Médicas. Universidad Privada del Guairá - UPG. Ciudad del Este, Paraguay.

*E-mail: elder.enf@hotmail.com

${ }^{4}$ Universidad de la Integración de las Américas. Asunción - Paraguay.

${ }^{5}$ Universidad Iberoamericana (UNIBE). Asunción - Paraguay.

SUBMETIDO EM: 3/2019 


\section{RESUMO}

Objetivos: Descrever a saúde ergonômica de professores de uma escola militar na região amazônica, visto que o trabalho realizado por esta causa interfere na integridade psicofisiológica do trabalhador. Método: Foi aplicado o instrumento de avaliação de fadiga utilizado pela Ergo Assessoria e Consultoria em Saúde do Trabalhador, validada por Couto, utilizado nas mais diversas práticas ocupacionais. Este instrumento mede o nível de desconformidade do professor no início e no final do horário de trabalho. Resultados: As maiores desconformidades apresentadas foram estresse 37\% (23), dor muscular 32\% (20) e fadiga 31\% (19). No início do dia de trabalho, as maiores inconformidades relatadas foram classificadas como fatores de intensidade um (1), caracterizadas pela ausência de dor nos braços ou punhos das mãos, ausência de dor nas costelas e produtividade normal. Ao final do dia, as maiores inconformidades relatadas foram fatores de intensidade de três (3) a quatro (4), como dor nas costelas, dor nas mãos e punhos do braço dominante e o fator nervoso. Conclusão: O processo de trabalho docente apresenta um risco ergonômico moderado, o trabalho é estressante e requer ajustes para melhorar a qualidade de vida e o trabalho docente da escola.

Palavras-chave: Ergonomia, Ensino, Análise ergonômica.

\section{INTRODUCCIÓN}

El avance de la tecnología viene proporcionando al mundo constantes transformaciones, principalmente en las empresas e industrias. Los cambios generados a partir de las innovaciones tecnológicas transforman el ambiente de trabajo, las cuales pueden originar riesgos para la salud del trabajador (FERREIRA MC, 2008).

Las actividades desarrolladas por los docentes; podrían constituir un factor de riesgo; por los esfuerzos repetitivos diarios que el mismo realiza dentro y fuera de las aulas. La postura corporal puede ser analizada por la ergonomía a través de aparatos ergonómicos con métodos específicos, como el Método de Couto y la herramienta RULA (COUTO HA, 2018).

Existen innumerables factores que interfieren en la calidad del trabajo docente y, consecuentemente, en la salud del profesor. Marques GFC (2010) describe las condiciones de trabajo, que apuntan a características específicas cualitativas y cuantitativas, dependiendo del tiempo de trabajo realizado o la exposición al ambiente de trabajo.

Según Lida (2005), la ergonomía estudia las características psicológicas y físicas del trabajador con su ambiente de trabajo. La ergonomía constituye una ciencia interdisciplinaria que pretende reducir los problemas de relación hombre-máquina mediante la adecuación del método o herramienta al operador.

La ausencia de ergonomía origina numerosos disturbios en el operador, siendo la principal, la osteomuscular. Estos disturbios, han despertado el interés de los investigadores, debido al alto costo y al impacto en la calidad de vida de los trabajadores (FERREIRA MC, 2008).

Según Ferreira (2008) las implicaciones económicas, políticas, tecnológicas y culturales del trabajo y sus diferentes actores (dirigentes, gestores, trabajadores y usuarios/clientes) fundamentan la importancia del debate sobre la calidad de vida en el trabajo y la ergonomía.

Según Chaffin DB, et al (2001) y Lida (2005), la ergonomía y la biomecánica ocupacional son complementarias entre sí, ya que la ergonomía busca perfeccionar el desempeño y la seguridad del trabajador a través del estudio de los movimientos y el desarrollo de principios generales que conducen a la interacción humana en el ambiente de trabajo.

La vulnerabilidad puede ser comprendida como un conjunto de factores que pueden aumentar o disminuir el riesgo a que estamos expuestos en todas las situaciones de nuestra vida, así como también como una forma de 
evaluar las probabilidades que cada persona tiene de contraer enfermedades, incluyendo las infecciosas (SANTOS JLG, 2012).

En la práctica, la evaluación ergonómica del trabajo es realizada a través de métodos, instrumentos y normas, que focalizan las condiciones de trabajo en un área específica (LAPERUTA DGP et al, 2014). El objetivo de este trabajo, fue describir la salud ergonómica de los docentes de una escuela militar en la región del Amazonas durante el año 2018.

\section{MÉTODOS}

La metodología utilizada fue la cualitativa. En una primera parte se realizó una exhaustiva revisión bibliográfica, investigación en libros, revistas y artículos científicos tanto en formato físico como en línea. El ámbito geográfico fue la ciudad de Manaus, Amazonas, Brasil, durante el período de septiembre a noviembre del 2018.

El trabajo de campo fue realizado en la Escuela Publica Militar del Estado de Amazonas. El plantel docente estuvo compuesto por 62 profesores, siendo 47 mujeres y 15 hombres, responsables de las disciplinas básicas de enseñanza infantil, fundamental de $6^{\circ}$ a $9^{\circ}$ año y de enseñanza media.

Para la recolección de datos, se solicitó la autorización del director de la escuela y el consentimiento del grupo docente. El instrumento fue presentado y explicado a los docentes, para que los mismos entiendan y para que los mismos aclararen todas sus dudas, las cuales fueron respondidas por el equipo investigador. El instrumento fue autoaplicativo y fue omitida la identificación de los participantes para mantener el anonimato de los mismos.

Los investigadores acompañaron a los docentes en las prácticas más diversas y estos fueron evaluados a través de los análisis ergonómicos en su ambiente de trabajo. Fue aplicado un instrumento de evaluación de fatiga utilizado por la Asesoría y Consultoría en Salud Ocupacional (ERGO), el cual es utilizado en las más diversas prácticas ocupacionales, siendo validado por Couto HA (2018).

Según Gomide ABL (2010), el cuestionario permite evaluar la sensación de los docentes en el momento en que es aplicado el instrumento. Fueron excluidos del trabajo, profesores con menos de dos meses de actividad docente, quienes poseían diagnóstico de enfermedades Lesiones por Esfuerzos Repetitivos (LER) y Disfunciones Osteomusculares Relacionadas al Trabajo (DORT).

A través de los movimientos practicados por el profesor, fue posible la aplicación de herramientas de análisis ergonómico las cuales fueron:

- Lista de chek list de Couto (2007); para la evaluación del riesgo en los miembros superiores.

- Instrumento RULA para la medición y evaluación de la exposición de las posturas, fortalezas y actividades musculares inadecuadas que contribuyen para el desarrollo del LER / DORT. La herramienta RULA fue desarrollada dentro de la ergonomía para realizar diagnósticos simples, pues para el desarrollo de sus análisis se usan tan solo lápiz y papel (ASSUNÇÃO AÁ, VILELA LVO, 2009).

En esta investigación fueron necesarios descomponer las actividades desarrolladas por los profesores en cinco (5) secuencias de movimientos, las cuales fueron suficientes para que el análisis ergonómico resultase en un diagnóstico del proceso del trabajo docente.

En la secuencia uno (1), se observa que el profesor realiza un movimiento de flexión del hombro derecho de $68,68^{\circ}$ al posicionar el marcador en la pizarra. Presenta una leve rotación interna del codo y del hombro derecho. Con la mano derecha realiza el movimiento de pinza al agarrar el marcador para escribir en la pizarra.

En la secuencia dos (2), se observa que el profesor realiza una rotación interna del codo asociado a una flexión del mismo. Con la mano derecha, el profesor agarra el marcador para la pizarra con un movimiento de 
pinza asociado a una leve extensión de la mano derecha, pudiendo generar molestias al finalizar la jornada de trabajo. Se observa también una leve flexión de la cervical.

En la secuencia tres (3), el profesor realiza un movimiento de flexión del codo. También presenta una leve extensión del puño derecho al escribir en la pizarra, pudiendo así resultar en un movimiento que puede generar molestias futuras. Presenta una flexión de la cervical asociada a una leve rotación de la misma. Se observa también una leve rotación del tronco.

En la secuencia cuatro (4), se observa que el profesor realiza un movimiento de flexión del codo asociada a una rotación interna del mismo. Realiza un movimiento de pinza para agarrar el marcador para la pizarra y también se observa una flexión del hombro de 89,07ํ․

En la secuencia cinco (5), el profesor ejecuta el movimiento de la pinza pulpar, al agarrar el borrador para la pizarra asociada a una extensión. Presenta también, el movimiento de flexión de $43,60^{\circ}$ de la cervical al mirar para el borrador.

Este estudio fue aprobado por el Comité de Ética en Investigación de la Universidad Estadual de Amazonas UEA, Manaus (AM) № 3.128.470, y CAAE 03140018.4.0000.5016.

\section{RESULTADOS Y DISCUSION}

La organización del trabajo es imprescindible para que una actividad sea productiva, eficiente y eficaz. Existen innumerables métodos e instrumentos que identifican situaciones que pueden ser perjudiciales para la salud y el buen desempeño del trabajador en su local de trabajo, pudiendo ser estas posturales, organizacionales o ambientales (SHIDA GJ, BENTO PEG, 2012).

En la aplicación del Check list de Couto, fue diagnosticado un factor biomecánico de moderada importancia, o sea, fue detectado un riesgo ergonómico que puede ocasionar molestias osteomusculares y enfermedades ocupacionales futuras a los docentes, en caso de que el riesgo ergonómico no sea tratado y mejorado. Con la aplicación de la herramienta RULA también fue diagnosticado un riesgo moderado, y de la misma manera, en caso de que el riesgo no sea tratado y mejorado, puede originar a los docentes molestias osteomusculares y enfermedades ocupacionales futuras (Tabla 1).

Tabla 1 - Resultado de la aplicación de las herramientas de análisis ergonómica.

\begin{tabular}{lll}
\hline Herramienta utilizada & Resultado & \\
\hline Chek List de Couto para la Evaluación de Riesgos de Disturbios en los & Factor biomecánico de moderada \\
Miembros Superiores & $\begin{array}{l}\text { importancia } \\
\text { información }\end{array}$ \\
RULA & inforado - investigar \\
\hline
\end{tabular}

Fuente: Gomes W, Oliveira da Silva E, dos Santos S, Ramos P, de Araujo E, Diascanio JM. La salud ergonómica del docente en una escuela militar en la región Amazónica. Brasil (2018).

Al realizar un análisis ergonómico es necesario tener conocimiento del espacio donde el trabajador ejerce su actividad, por lo que también es necesario realizar una medición de su espacio de trabajo (Tabla 2).

Tabla 2 - Medición del espacio de trabajo docente.

\begin{tabular}{ll}
\hline Dimensiones de la pizarra & $1,40 \mathrm{~cm} \times 1,00 \mathrm{~cm}$ (dos pizarras por sala de aula). \\
Altura de la pizarra & $80 \mathrm{~cm}$ del piso. \\
Distancia del profesor hasta la pizarra & Aproximadamente $50 \mathrm{~cm}$.
\end{tabular}

Fuente: Gomes W, Oliveira da Silva E, dos Santos S, Ramos P, de Araujo E, Diascanio JM. La salud ergonómica del docente en una escuela militar en la región Amazónica. Brasil (2018). 
Los profesores fueron analizados y acompañados por los investigadores y especialistas en ingeniería de seguridad del trabajo. En el presente estudio, los profesores dictaban clases aproximadamente 8 horas por día, utilizando como herramienta principal el marcador para pizarra y realizando movimientos repetitivos durante toda su jornada de trabajo.

Fueron relatadas a los investigadores molestias osteomusculares y quejas de cansancio al final de la clase. Así, fue posible conocer aquellos movimientos con mayor impacto sobre en su jornada de trabajo. El estrés fue referido por el 37\% (23) de los docentes, siendo esta la más prevalente (19) (Tabla 3).

Tabla 3 - Molestias más frecuentes referidas por los docentes.

\begin{tabular}{|c|c|c|}
\hline Molestias & Frecuencia & Porcentaje \\
\hline Estrés & 23 & 37 \\
\hline Dolores musculares & 20 & 32 \\
\hline Fatiga & 19 & 31 \\
\hline TOTAL & 62 & 100 \\
\hline
\end{tabular}

Fuente: Gomes W, Oliveira da Silva E, dos Santos S, Ramos P, de Araujo E, Diascanio JM. La salud ergonómica del docente en una escuela militar en la región Amazónica. Brasil (2018).

Se observo que en el grupo docente existieron molestias de intensidad que variaba entre uno (1) y tres (3). Destacándose en la intensidad uno (1) de molestia la ausencia de dolores en los brazos o puños de las manos, ausencia de dolor en las costillas y con productividad normal.

Para las molestias de intensidad dos (2), se destacaron los factores: descansado, ausencia de dolores en las piernas y buena concentración por haber presentado una media de 7 marcaciones entre el grupo docente.

En la intensidad tres (3) se destacaron los factores de descansado, ausencia de dolor en los pies y descansado visualmente por tener el mayor índice de ocurrencia en el grupo docente, aun siendo el inicio de la jornada.

De esta manera, los docentes presentaban, aun en el inicio de la jornada de trabajo, algún tipo de queja, que afectaban directamente el desempeño y la productividad durante el día de trabajo.

En una investigación realizada por Carvalho AJFP \& Alexandre NMC (2006), en escuelas de enseñanza fundamental los profesores presentaron una mayor frecuencia de síntomas osteomusculares principalmente en las regiones lumbar $(63,1 \%)$, torácica $(62,4 \%)$, cervical $(59,2 \%)$, hombros $(58,0 \%)$, puños y manos $(43,9 \%)$. En relación a la prevalencia en los últimos 7 días, las áreas corporales más citadas fueron hombros (29,9\%), región cervical $(28,7 \%)$, lumbar $(27,4 \%)$, torácica $(27,4 \%)$, puños y manos $(14,6 \%)$. Por lo tanto, la mayor frecuencia de síntomas osteomusculares estuvo presente en todas las regiones del área corpórea, destacándose factores de riesgos para la vida y para el ambiente de trabajo.

Se observó que el grupo docente presentó diversas intensidades de molestias, principalmente entre los rangos de tres (3) a seis (6) intensidades. Al término de la jornada de trabajo los docentes presentaban mayores quejas debido a estas molestias.

Entre las quejas más significativas expresadas por los docentes al final del día, se destacaron las molestias con rangos de intensidad de tres (3) a cuatro (4), tales como dolores en las costillas, en las manos y puños del brazo dominante y el factor nervioso.

Los dolores en las costillas, la dificultad para concentrarse, el cansancio visual, la productividad comprometida y los dolores en los pies, fueron los factores de mayor destaque entre las molestias mencionadas por los docentes. Los grupos docentes reconocen estar cansados y estresados al término de su jornada laboral, con una gran intensidad (intensidad de 5).

REAS/EJCH | Vol. 11 (11) | e447 | DOI: https://doi.org/10.25248/reas.e447.2019 Página 5 de 8 
Con mayor intensidad de molestias, los factores que más afectan a los docentes, al término de su jornada laboral fueron; cansancio, dolor del musculo del cuello y hombro, dolores de cabeza y en las piernas.

Hall (2005) describe los movimientos humanos como los constituidos por movimientos generales, los cuales incluyen simultáneamente la translación y rotación. Esta constituye una combinación compleja de componentes de movimientos lineales, donde todas las partes del cuerpo se mueven en una misma dirección y con la misma velocidad. Los movimientos angulares realizados son de rotación, ya que estos se realizan alrededor de un punto central.

De esta manera, se evidencia que el grupo docente manifiesta molestias a lo largo de la jornada de trabajo, y al término de la misma estas se presentan con mayores intensidades, comprometiendo no solamente su calidad de vida en el trabajo, sino también la salud en su vida personal. Los resultados aquí presentados, representan la percepción del equipo de docentes, vivencias y expectativas en su local de trabajo. Respecto a la ergonomía, el proceso de trabajo docente presento riesgos ergonómicos, lo que hace necesario realizar alteraciones en el ambiente de trabajo del profesor.

En relación a la organización del trabajo, el profesor dispone de intervalos de 30 minutos durante su jornada de trabajo para el receso. Tener poco tiempo para el descanso se torna perjudicial para la salud del mismo, llevando al cansancio rápidamente, aumentando la probabilidad de estrés (ALVES G, 2011). Estas probabilidades varían y son dependientes tanto de factores biológicos como sociales y culturales. Por tanto, se incluyen aquellos ambientes relacionados al trabajo, así como los relacionados a los profesionales (SANTOS JLG, 2012).

Como resultado de esta investigación, se presentan algunas recomendaciones dirigidas a todo el grupo docente y a la dirección de las escuelas, para mejorar el proceso del trabajo y que beneficien a los profesores, a la escuela y a los alumnos (Tabla 4).

Tabla 4 - Recomendaciones para las mejorías conforme a la NR 17.

RECOMENDACIONES

Implementación de retroproyectores en cada sala de aula

Métodos activos de enseñanza

Concientización de los docentes en cuanto a los riesgos ergonómicos

\section{OBJETIVO}

Eliminar los riesgos de molestias osteomusculares debido a la excesiva realización de movimientos biomecánicos de los miembros superiores

Reducir el uso de la pizarra al dar clases

Disminuir los riesgos posturales de los docentes

\section{ADECUACIÓN A LA NORMA NR17}

17.4. Equipamientos de puestos de trabajo 17.4.1. Todos los equipamientos que componen un puesto de trabajo deben estar adecuados a las características psicofisiológicas de los trabajadores y estar de acuerdo a la naturaleza del trabajo a ser realizado

17.6.1. La organización del trabajo debe ser adecuada a las características psicofisiológicas de los trabajadores y estar de acuerdo a la naturaleza del trabajo a ser realizado

Art. 157 dada por la Ley No 6.514 de 22.12.1977 (BRASIL, 1977). Cabe a las empresas....II. Instruir a los empleados, a través de ordenes de servicio, en cuanto a las precauciones a tomar para evitar los accidentes de trabajo o enfermedades ocupacionales.

Fuente: Gomes W, Oliveira da Silva E, dos Santos S, Ramos P, de Araujo E, Diascanio JM. La salud ergonómica del docente en una escuela militar en la región Amazónica. Brasil (2018). 
El equipo docente de la escuela posee poco conocimiento acerca de cómo mejorar el ambiente de trabajo. Por esto, se recomienda a la escuela, la concientización de los docentes en cuanto a los riesgos ergonómicos y lo que deben hacer para disminuir los riesgos posturales.

En la escuela militar donde fue realizada esta investigación, existía una organización de trabajo definida por la gestión de policías militares y por el grupo pedagógico de los profesores de la secretaria del Estado de Educación y Calidad de la Enseñanza (SEDUC/AM) del Estado del Amazonas. Es muy importante conocer la organización escolar, para promover el diagnostico ergonómico del proceso de trabajo docente.

Para realizar un diagnóstico ergonómico, es esencial describir en detalles la actividad desarrollada por el profesor. Las actividades que los profesores generalmente realizan son; enseñar a los alumnos basados en su plan diario, escribir en su cuaderno las actividades, tomar el marcador para escribir en la pizarra, escribir en la esquina superior izquierda de la pizarra, escribir el contenido del programa. El profesor se da vuelta para los alumnos y explica el contenido y las actividades son iniciadas nuevamente hasta el final de la clase o hasta que todo el plan programado de clase sea realizado. Es importante describir el método de trabajo utilizado por los docentes de la escuela, consistentes en actividades realizadas de pie, actividades predominantemente de los miembros superiores, principalmente en el lado dominante, con carga efectiva de trabajo de 8 horas, con intervalos de 2 horas para el almuerzo o para ir a los sanitarios.

\section{CONCLUSIÓN}

Se concluye que el proceso de trabajo docente presenta un riesgo ergonómico moderado, y que el trabajo de los docentes es estresante y cansador. Es necesario realizar adecuaciones para mejorar la calidad de vida y el trabajo docente.

\section{REFERENCIAS}

1. ABERGO - Associação Brasileira de Ergonomia. Disponível em:<http://www.abergo.org.br/>. Acesso em: 3 set. 2018.

2. ALVES G. Trabalho e subjetividade: o espírito do toyotismo na era do capitalismo manipulatório. São Paulo: Boitempo, 2011.

3. ASSUNÇÃO AÁ, VILELA LVO. Lesões por esforços repetitivos: guia para profissionais de saúde. Piracicaba - SP: Centro de Referência em Saúde do Trabalhador - CEREST, 2009.

4. CARVALHO AJFP, ALEXANDRE NMC. Sintomas Osteomusculares em Professores do Ensino Fundamental. Revista Brasileira de Fisioterapia, Campinas, SP, Vol. 10, No. 1 (2006), 35-41.

5. CHAFFIN DB, ANDERSSON GBJ, MARTIN BJ, Biomecânica Ocupacional. Belo Horizonte: Ergo, 2001.

6. COUTO HA. Gerenciando a LER e as DORT nos tempos atuais. Belo Horizonte: Ergo Ltda, 2007.

7. COUTO HA. Ferramentas Ergonômicas. Disponível em: <http://ergoltda.com.br/checklist/index.html >. Acesso em: 21 out. 2018.

8. Ergo: Assessoria e consultoria saúde ocupacional http://www.ergoltda.com.br/downloads/questionario_bipolar_ava_fadiga. Acesso em 12 de set. 2018.

9. FALCÃO FS. Métodos de Avaliação Biomecânica Aplicados A Postos De Trabalho No Pólo Industrial De Manaus (Am): Uma Contribuição Para O Design Ergonômico. 2007. Dissertação de mestrado - Programa de Pós-Graduação em Desenho Industrial da Universidade Estadual Paulista Júlio de Mesquita Filho; Bauru, SP. 2007.

10. FERREIRA MC. A ergonomia da atividade se interessa pela qualidade de vida no trabalho: Reflexões empíricas e teóricas. Cad. psicol. soc. trab. [online]. 2008, vol.11, n.1, pp. 83-99. ISSN 1516-3717.

11. GOMIDE ABL. Análise da carga física de trabalho para prevenção da fadiga - um estudo de caso: operador de checkouts. 2010. 53. Dissertação (Mestrado em Design) - Universidade Estadual Paulista, Bauru, 2010.

12. HALL SJ. Biomecânica Básica. $4^{a}$ ed. Rio de Janeiro, RJ. Editora. Guanabara Koonga S.A, 2005.

13. LIDA I. Ergonomia, projeto e produção. 2.ed. rev. e ampl. São Paulo: Edgard Blücher, 2005.

REAS/EJCH | Vol. 11 (11) | e447 | DOI: https://doi.org/10.25248/reas.e447.2019 Página 7 de 8 
14. LAPERUTA DGP et al. Análise crítica de Métodos/Ferramentas de Avaliação Ergonômica: perspectivas para novas abordagens. A Journal of Prevention, Assessment, and Rehabilitation, ISSN 1051-9815 (impresso), Qualis 2014 Engenharias III: B2.

15. LIGEIRO, J. Ferramentas de avaliação ergonômica em atividades multifuncionais: a contribuição da ergonomia para o design de ambientes de trabalho. 2010. 219 f. Dissertação (Pós-Graduação em Design), Programa de Pós-Graduação em Design - Universidade Estadual Paulista Júlio de Mesquita Filho, 2010.

16. MARQUES GFC. As condições do trabalho docente e o processo ensino-aprendizagem nos anos iniciais do ensino fundamental. 2010. 110 f. Dissertação (Pós-Graduação em Educação), Programa de Pós-Graduação em Educação Universidade Federal de Juiz de Fora, 2010.

17. SEDUC- Secretria de Estado de Educação e Qualidade de Ensino. www.seduc.am.gov.br/ acesso em 18 set. 2018.

18. SHIDA GJ, BENTO PEG. Métodos e ferramentas ergonômicas que auxiliam na análise de situações de trabalho. Congresso Nacional de Excelência em Gestão, 8. 2012, Rio de Janeiro, RJ.

19. SANTOS JLG et al. Risco e vulnerabilidade nas práticas dos profissionais de saúde. Rev. Gaúcha Enferm., Porto

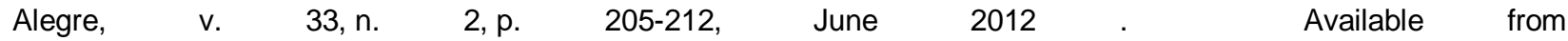
<http://www.scielo.br/scielo.php?script=sci_arttext\&pid=S1983-14472012000200028\&lng=en\&nrm=iso>. access on 08 Apr. 2019. http://dx.doi.org/10.1590/S1983-14472012000200028. 